\title{
States, Symmetries and Superselection
}

\author{
Domenico Giulini \\ Theoretische Physik, Universität Zürich, \\ Winterthurerstrasse 190, CH-8057 Zürich, Switzerland
}

\begin{abstract}
The process of dynamical decoherence may cause apparent superselection rules, which are sometimes called 'environmentally induced' or 'soft'. A natural question is whether such dynamical processes are eventually also responsible for at least some of the superselection rules which are usually presented as fundamentally rooted in the kinematical structure of the theory (so called 'hard' superselection rules). With this question in mind, I re-investigate two well known examples where superselection rules are usually argued to rigorously exist within the given mathematical framework. These are (1) the Bargmann superselection rule for the total mass in Galilei invariant quantum mechanics and (2) the charge superselection rule in quantum electrodynamics. I argue that, for various reasons, the kinematical arguments usually given are not physically convincing unless they are based on an underlying dynamical process.
\end{abstract}

\section{Introduction}

Fundamental to the concept of dynamical decoherence is the notion of 'delocalization' 12] [8]. The intuitive idea behind this is that through some dynamical process certain state characteristics ('phase relations'), which were locally accessible at one time, cease to be locally accessible in the course of the dynamical evolution. Hence locally certain superpositions cannot be verified anymore and an apparent obstruction to the superposition principle results. Such mechanisms can therefore be considered responsible for so-called soft superselection rules, like that of molecular chirality [18]. They are called 'soft', because they only hold with respect to the limited class of local observables and are hence of approximate validity. But there are also 'hard' superselection rules, which are usually presented as rigorous mathematical results within the kinematical framework of the theory. Such presentations seem to suggest that there is no room left for a dynamical interpretation, and that hence these two notions of superselection rules are really distinct. Here I wish to argue that at least some of the existing proofs for 'hard' superselection rules give a false impression, and that quite to the contrary they actually need some dynamical input in order to be physically convincing. We will look at the case of Bargmann's superselection rule for total mass in ordinary quantum mechanics and that of charge in quantum electrodynamics (QED). The discussion of the latter will be heuristic insofar as we will pretend that QED is nothing but quantum mechanics (in the Schrödinger 
representation) of the infinite-dimensional (constrained) Hamiltonian system given by classical electrodynamics.

Crucial to the ideas presented here is of course that 'delocalized' does not at all mean 'destroyed', and that hence the loss of quantum coherence is only an apparent one. This distinction might be considered irrelevant FAPP but it is important in attempts to understand apparent losses of quantum coherence within the standard dynamical framework of quantum mechanics.

As used here, the term 'local' usually refers to locality in the (classical) configuration space $Q$ of the system, where we think of quantum states in the Schrödinger representation, i.e., as $L^{2}$-functions on $Q$. Every parametrization of $Q$ then defines a partition into 'degrees of freedom'. Locality in $Q$ is a more general concept than locality in ordinary physical space, although the latter forms a particular and physically important special case. Moreover, on a slightly more abstract level, one realizes that the most general description of why decoherence appears to occur is that only a restricted set of so-called physical observables are at ones disposal, and that with respect to those the relevant 'phase relations' apparently fade out of existence. It is sometimes convenient to express this by saying that decoherence occurs only with respect (or relative) to a 'choice' of observables. Clearly this 'choice' is not meant to be completely free, since it has to be compatible with the dynamical laws and the physically realizable couplings (compare 12]). But to fully control those is a formidable task - to put it mildly - and a careful a priori characterization of observables seems therefore almost always inescapable. In this respect the situation bears certain similarities to that of 'relevant' and 'irrelevant' degrees of freedom in statistical mechanics.

\section{Hilbert Spaces and Observables}

The mathematical modelling of a physical system involves a specification of a space of states and a space of observables. In quantum theory this is usually done with the help of the underlying classical theory. States are then identified with the space of rays in the Hilbert space $\mathcal{H}=L^{2}(Q)$, which we denote by $\mathcal{P H}$ (projective Hilbert space) and observables are certain operators on $\mathcal{H}$. I am not aware of any generally valid criteria by which one might fully characterize sets of operators on $\mathcal{H}$ 'as physical observables'. But there are certain mathematical structures which seem physically admissible and also natural, which, once imposed, allow to make some general statements about the set of physical observables.

Self-Adjointness is a generally accepted criterion, and without loss of generality one can also restrict to bounded operators. Although only certain combinations of addition and multiplication preserve self-adjointness, it is mathematically more convenient to consider the whole algebra generated (in a sense made more precise below) by this set. This algebra is then called the algebra

\footnotetext{
${ }^{1}$ For All Practical Purposes.
} 
of physical observables, $\mathcal{O}$, although only its self-adjoint elements actually correspond to observables. Moreover, since physically the matrix elements are the relevant quantities, it is natural to require that a sequence of operators converges if and only if (henceforth: iff) all the matrix elements converge; in technical terms, the algebra should be (topologically) closed in the weak operator topology, i.e., be a von Neumann algebra. An extremely useful fact is that weak closures of algebras can be characterized in purely algebraic terms. This works as follows: Let $\mathcal{B}(\mathcal{H})$ denote the algebra of bounded operators on $\mathcal{H}$. If $\mathcal{A} \subseteq \mathcal{B}(\mathcal{H})$ is any subset, then $\mathcal{A}^{\prime}:=\{B \in \mathcal{B}(\mathcal{H}): A B=B A, \forall A \in \mathcal{A}\}$ is called the commutant of $\mathcal{A}$. Iterating this procedure leads to $\mathcal{A}^{\prime \prime}$, of which the following is true: (i) $\mathcal{A}^{\prime \prime}$ is a von Neumann algebra, (ii) it is the smallest von Neumann algebra containing $\mathcal{A}$. In this sense one says that $\mathcal{A}^{\prime \prime}$ is the von Neumann algebra generated by $\mathcal{A}$. In particular, if $\mathcal{A}$ was already a von Neumann algebra, it must satisfy $\mathcal{A}=\mathcal{A}^{\prime \prime}$. Note also that, by definition of the commutant, an inclusion of the form $\mathcal{A} \subseteq \mathcal{B}$ implies $\mathcal{B}^{\prime} \subseteq \mathcal{A}^{\prime}$.

Recall that the center, $\mathcal{O}^{c}$, of $\mathcal{O}$ consists of those elements in $\mathcal{O}$ which also lie in the commutant $\mathcal{O}^{\prime}$, i.e., commute with all elements in $\mathcal{O}$; hence $\mathcal{O}^{c}:=\mathcal{O} \cap \mathcal{O}^{\prime}$. Complex multiples of the identity operator are trivially contained in $\mathcal{O}^{c}$ and any other ones are called superselection operators. If $\mathcal{O}^{c}$ contains a projection operator onto a subspace $\mathcal{H}^{\prime} \subset \mathcal{H}$, then $\mathcal{O}$ must leave $\mathcal{H}^{\prime}$ invariant and $\mathcal{H}^{\prime}$ is said to reduce $\mathcal{O}$. In this case the theory is said to possess superselection rules. In the opposite case, $\mathcal{O}$ acts irreducibly on $\mathcal{H}$ and no superselection rules exist.

We now list some simple and general criteria which have been suggested in the literature to characterize $\mathcal{O}$. These criteria will involve $\mathcal{O}$ and $\mathcal{O}^{\prime}$ and hence do not only concern the abstract algebraic object $\mathcal{O}$, but also $\mathcal{H}$ on which $\mathcal{O}^{\prime}$ depends. Hence they can either be read as condition on $\mathcal{O} \subseteq \mathcal{B}(\mathcal{H})$ given $\mathcal{H}$, or as certain 'matching conditions' between the two mathematical objects representing physical observables on one hand and states on the other.

- In 1932, von Neumann [14 proposed to identify $\mathcal{O}=B(\mathcal{H})$. Then $\mathcal{O}^{\prime}=$ $\{\alpha \mathbf{1}, \alpha \in \mathbf{C}\}$ and hence no superselection rules exist.

- In 1952, Wick, Wightman, and Wigner 17 considered for the first time the possibility that $\mathcal{O}$ might be strictly smaller than $B(\mathcal{H})$ and not act irreducibly on $\mathcal{H}$, so that $\mathcal{H}=\oplus_{i} \mathcal{H}_{i}$, where each $\mathcal{H}_{i}$ reduces $\mathcal{O}$. The projectors onto the $\mathcal{H}_{i}$ obviously lie in $\mathcal{O}^{c}$ and hence are superselection operators. Now only the rays in $\bigcup_{i} \mathcal{H}_{i}$ define pure states. This can be generalized to direct integrals.

- In 1960, Jauch [10] properly formulated Dirac's condition, that there should exist at least one 'complete set of commuting observables', which in this formulation makes sense only for operators with discrete spectra. The generally valid formulation is, that $\mathcal{O}$ should contain a von Neumann subalgebra $\mathcal{A} \subseteq \mathcal{O}$ which is maximal abelian. Being abelian clearly means that $\mathcal{A} \subseteq \mathcal{A}^{\prime}$ and being maximal means that everything that commutes with $\mathcal{A}$ is already contained in it, i.e., $\mathcal{A}^{\prime} \subseteq \mathcal{A}$. Taken together, maximal abelian is equivalent to $\mathcal{A}=\mathcal{A}^{\prime}$. 
This condition can be read as saying that $\mathcal{O}$ cannot be too small, since it must accommodate an abelian subalgebra that is maximal.2] Now, the point is that the existence of a maximal abelian $\mathcal{A} \subseteq \mathcal{O}$ can be equivalently expressed just in terms of $\mathcal{O}$ and $\mathcal{O}^{\prime}$, namely by saying that everything that commutes with $\mathcal{O}$ is contained in $\mathcal{O}$, that is, $\mathcal{O}^{\prime} \subseteq \mathcal{O}$ (Dirac-Jauch condition). Necessity of this condition is readily seen, since $\mathcal{A} \subseteq \mathcal{O}$ implies $\mathcal{O}^{\prime} \subseteq \mathcal{A}^{\prime}=\mathcal{A} \subseteq \mathcal{O}$. The converse was shown by Jauch [10]. Note also that $\mathcal{O}^{c}:=\mathcal{O} \cap \mathcal{O}^{\prime}$ implies that $\mathcal{O}^{\prime} \subseteq \mathcal{O}$ can be rewritten as $\mathcal{O}^{c}=\mathcal{O}^{\prime}$, which essentially says that the center of $\mathcal{O}$ already exhausts the set of all those elements in $\mathcal{B}(\mathcal{H})$ commuting with $\mathcal{O}$.

- In 1961, Jauch and Misra 11 discussed the relation between superselection rules and gauge symmetries (supersymmetries in their language). Since by definition gauge symmetries commute with physical observables, they are generated by the unitary elements of $\mathcal{O}^{\prime}$. But since $\mathcal{O}$ is a von Neumann algebra, we have $\mathcal{O}=\mathcal{O}^{\prime \prime}$. Therefore $\mathcal{O}^{\prime} \subseteq \mathcal{O}$ is equivalent to $\mathcal{O}^{\prime} \subseteq \mathcal{O}^{\prime \prime}$, i.e. that $\mathcal{O}^{\prime}$ is abelian. The Dirac-Jauch condition is therefore equivalent to the requirement that gauge groups should be abelian.3

\section{Superselection Rules via Symmetry Requirements}

The requirement that a certain group must act on the set of all physical states is often the (kinematical) source of superselection rules. Here I wish to explain the structure of this argument.

Note first that in quantum mechanics we identify the states of a closed system with rays and not with vectors which represent them (in a redundant fashion). It is therefore not necessary to require that a symmetry group $G$ acts on the Hilbert space $\mathcal{H}$, but rather it is sufficient that it acts on $\mathcal{P} \mathcal{H}$,

${ }^{2}$ Without further qualification the term 'maximal' always means 'maximal in $\mathcal{B}(\mathcal{H})^{\prime}$. In contrast, $\mathcal{A} \subseteq \mathcal{O}$ is said to be maximal abelian in $\mathcal{O}$ if $\mathcal{A}=\mathcal{A}^{\prime} \cap \mathcal{O}$. It would be pointless to require maximality in $\mathcal{O}$, since such subalgebras always exist (by Zorn's Lemma). Obviously maximality in $\mathcal{B}(\mathcal{H})$ implies maximality in $\mathcal{O}$. But in the general case the converse is true iff the Dirac-Jauch condition $\mathcal{O}^{\prime} \subseteq \mathcal{O}$ is met. Proof: Suppose (a) $\mathcal{O}^{\prime} \subseteq \mathcal{O}$, (b) $\mathcal{O}^{\prime} \subseteq \mathcal{A}^{\prime}$ (obvious from $\mathcal{A} \subseteq \mathcal{O}$ ), and (c) $\mathcal{A}=\mathcal{A}^{\prime} \cap \mathcal{O}$. Then for $Z \in \mathcal{O}^{\prime}$ (a), (b) and (c) immediately imply $Z \in \mathcal{A}$, hence $\mathcal{O}^{\prime} \subseteq \mathcal{A}$ or $\mathcal{A}^{\prime} \subseteq \mathcal{O}^{\prime \prime}=\mathcal{O}$. Then (c) is equivalent to $\mathcal{A}=\mathcal{A}^{\prime}$.

3 Note that the statement is not that gauge symmetries need a priori be abelian. For example, the permutation group for $n$ particles $(n>2)$ is perfectly legitimate to start with. However, the unreduced $n$-particle Hilbert space is now definitely too big in the sense that it contains higher-dimensional subspaces in which each ray defines the same pure state. Such a redundancy is against the Dirac-Jauch condition, which in particular implies that $\mathcal{O}$ should separate the rays in the space of physical states. In this case the Dirac-Jauch condition is met by truncating $\mathcal{H}$ so as to leave only one ray per pure state. Thereby the original gauge group is broken down to a residual one which is abelian. See [6]. 
the space of rays, via so-called ray-representations. Mathematically this is a non-trivial relaxation since not every ray-representation of a symmetry group $G$ (i.e. preserving the ray products) lifts to a unitary action of $G$ on $\mathcal{H}$. What may go wrong is not that for a given $g \in G$ we cannot find a unitary (or anti-unitary) operator $\mathrm{U}_{g}$ on $\mathcal{H}$; that is assured by Wigner's theorem (see [i] for a proof). Rather, what may fail to be possible is that we can choose the $\mathrm{U}_{g}$ 's in such a way that we have an action, i.e., that $\mathrm{U}_{g_{1}} \mathrm{U}_{g_{2}}=U_{g_{1} g_{2}}$. As is well known, this is precisely what happens for the implementation of the Galilei group in ordinary quantum mechanics. Without the admission of ray representations we would not be able to say that ordinary quantum mechanics is Galilei invariant.

To be more precise, to have a ray-representation means that for each $g \in G$ there is a unitary transformation $\mathrm{U}_{g}$ which, instead of the usual representation property, are only required to satisfy the weaker condition

$$
\mathrm{U}_{g_{1}} \mathrm{U}_{g_{2}}=\exp \left(i \xi\left(g_{1}, g_{2}\right)\right) \mathrm{U}_{g_{1} g_{2}}
$$

for some function $\xi: G \times G \rightarrow \mathbf{R}$, called multiplier exponent, satisfyingโ

$$
\begin{aligned}
\xi(1, g)=\xi(g, 1) & =0 \\
\xi\left(g_{1}, g_{2}\right)-\xi\left(g_{1}, g_{2} g_{3}\right)+\xi\left(g_{1} g_{2}, g_{3}\right)-\xi\left(g_{2}, g_{3}\right) & =0 .
\end{aligned}
$$

The second of these conditions is a direct consequence of associativity: $\mathrm{U}_{g_{1}}\left(\mathrm{U}_{g_{2}} \mathrm{U}_{g_{3}}\right)=\left(\mathrm{U}_{g_{1}} \mathrm{U}_{g_{2}}\right) \mathrm{U}_{g_{3}}$. Obviously these maps project to an action of $G$ on $\mathcal{P H}$. Any other lift of this action on $\mathcal{P H}$ onto $\mathcal{H}$ is given by a redefinition $\mathrm{U}_{g} \rightarrow \mathrm{U}_{g}^{\prime}:=\exp (i \gamma(g)) \mathrm{U}_{g}$, for some function $\gamma: G \rightarrow \mathbf{R}$ with $\gamma(1)=0$, resulting in new multiplier exponents

$$
\xi^{\prime}\left(g_{1}, g_{2}\right)=\xi\left(g_{1}, g_{2}\right)+\gamma\left(g_{1}\right)-\gamma\left(g_{1} g_{2}\right)+\gamma\left(g_{2}\right),
$$

which again satisfy (2) and (3). The ray representations $U$ and $U^{\prime}$ are then said to be equivalent, since the projected actions on $\mathcal{P H}$ are the same. We shall also say that two multiplier exponents $\xi, \xi^{\prime}$ are equivalent if they satisfy (㺼 for some $\gamma$.

We shall now see how the existence of inequivalent multiplier exponents together with the requirement that the group should act on the space of physical states, may clash with the superposition principle and thus give rise to superselection rules. For this we start from two Hilbert spaces $\mathcal{H}^{\prime}$ and $\mathcal{H}^{\prime \prime}$ and actions of a symmetry group $G$ on $\mathcal{P} \mathcal{H}^{\prime}$ and $\mathcal{P} \mathcal{H}^{\prime \prime}$, i.e., ray representations $\mathrm{U}^{\prime}$ and $\mathrm{U}^{\prime \prime}$ on $\mathcal{H}^{\prime}$ and $\mathcal{H}^{\prime \prime}$ up to equivalences (何). We consider $\mathcal{H}=\mathcal{H}^{\prime} \oplus \mathcal{H}^{\prime \prime}$

\footnotetext{
${ }^{4}$ For simplicity we ignore anti-unitary transformations. They cannot arise if, for example, $G$ is connected.

${ }^{5}$ The following conditions might seem a little too strong, since it would be sufficient to require the equalities in (2) and (3) only $\bmod 2 \pi$; this also applies to (国). But for our application in section 4 it is more convenient to work with strict equalities, which in fact implies no loss of generality.
} 
and ask under what conditions does there exist an action of $G$ on $\mathcal{P} \mathcal{H}$ which restricts to the given actions on the subsets $\mathcal{P} \mathcal{H}^{\prime}$ and $\mathcal{P} \mathcal{H}^{\prime \prime}$. Equivalently: when is $\mathrm{U}=\mathrm{U}^{\prime} \oplus \mathrm{U}^{\prime \prime}$ a ray representation of $G$ on $\mathcal{H}$ for some choice of ray-representations $U^{\prime}$ and $U^{\prime \prime}$ within their equivalence class? To answer this question, we consider

$$
\begin{aligned}
\mathrm{U}_{g_{1}} \mathrm{U}_{g_{2}} & =\left(\mathrm{U}_{g_{1}}^{\prime} \oplus \mathrm{U}_{g_{1}}^{\prime \prime}\right)\left(\mathrm{U}_{g_{2}}^{\prime} \oplus \mathrm{U}_{g_{2}}^{\prime \prime}\right) \\
& =\exp \left(i \xi^{\prime}\left(g_{1}, g_{2}\right)\right) \mathrm{U}_{g_{1} g_{2}}^{\prime} \oplus \exp \left(\xi^{\prime \prime}\left(g_{1}, g_{2}\right)\right) \mathrm{U}_{g_{1} g_{2}}^{\prime \prime}
\end{aligned}
$$

and note that this can be written in the form (11), for some choice of $\xi^{\prime}, \xi^{\prime \prime}$ within their equivalence class, iff the phase factors can be made to coincide, that is, iff $\xi^{\prime}$ and $\xi^{\prime \prime}$ are equivalent. This shows that there exists a ray-representation on $\mathcal{H}$ which restricts to the given equivalence classes of given ray representations on $\mathcal{H}^{\prime}$ and $\mathcal{H}^{\prime}$, iff the multiplier exponents of the latter are equivalent. Hence, if the multiplier exponents $\xi^{\prime}$ and $\xi^{\prime \prime}$ are not equivalent, the action of $G$ cannot be extended beyond the disjoint union $\mathcal{P} \mathcal{H}^{\prime} \cup \mathcal{P} \mathcal{H}^{\prime \prime}$. Conversely, if we require that the space of physical states must support an action of $G$ then non-trivial superpositions of states in $\mathcal{H}^{\prime}$ and $\mathcal{H}^{\prime \prime}$ must be excluded from the space of (pure) physical states.

This argument shows that if we insist of implementing $G$ as symmetry group, superselection rules are sometimes unavoidable. A formal trick to avoid them would be not to require $G$, but a slightly larger group, $\bar{G}$, to act on the space of physical states. $\bar{G}$ is chosen to be the group whose elements we label by $(\theta, g)$, where $\theta \in \mathbf{R}$, and the multiplication law is

$$
\bar{g}_{1} \bar{g}_{2}=\left(\theta_{1}, g_{1}\right)\left(\theta_{2}, g_{2}\right)=\left(\theta_{1}+\theta_{2}+\xi\left(g_{1}, g_{2}\right), g_{1} g_{2}\right) .
$$

It is easy to check that the elements of the form $(\theta, 1)$ lie in the center of $\bar{G}$ and form a normal subgroup $\cong \mathbf{R}$ which we call $Z$. Hence $\bar{G} / Z=G$ but $G$ need not be a subgroup of $\bar{G} \cdot \bar{G}$ is a central $\mathbf{R}$ extension ${ }^{6}$ of $G$ (see e.g. [15). Now a ray-representation $\mathrm{U}$ of $G$ on $\mathcal{H}$ defines a proper representation $\bar{U}$ of $\bar{G}$ on $\mathcal{H}$ by setting $U_{(\theta, g)}:=\exp (i \theta) \mathrm{U}_{g}$. Then $\bar{G}$ is properly represented on $\mathcal{H}^{\prime}$ and $\mathcal{H}^{\prime \prime}$ and hence also on $\mathcal{H}=\mathcal{H}^{\prime} \oplus \mathcal{H}^{\prime \prime}$. The above phenomenon is mirrored here by the fact that $Z$ acts trivially on $\mathcal{P} \mathcal{H}^{\prime}$ and $\mathcal{P} \mathcal{H}^{\prime \prime}$ but nontrivially on $\mathcal{P} \mathcal{H}$, and the superselection structure comes about by requiring physical states to be fixed points of $Z$ 's action.

\section{Test Case: Bargmann's Superselection Rule}

An often mentioned textbook example where a particular implementation of a symmetry group allegedly clashes with the superposition principle, such

\footnotetext{
${ }^{6}$ Had we defined the multiplier exponents $\bmod 2 \pi$ (compare footnote 5 ) then we would have obtained a $U(1)$ extension, which would suffice so far. But in the next section we will definitively need the $\mathbf{R}$ extension as symmetry group of the extended classical model discussed there.
} 
that a superselection rule results, is Galilei invariant quantum mechanics (e.g. [3]; see also Wightman's review [19]). We will discuss this example in detail for the general multi-particle case. (Textbook discussions usually restrict to one particle, which, due to Galilei invariance, must necessarily be free.) It will serve as a test case to illustrate the argument of the previous chapter and also to formulate my critique. Its physical significance is limited by the fact that the particular feature of the Galilei group that is responsible for the existence of the mass superselection rule ceases to exist if we replace the Galilei group by the Poincaré group (i.e. it is unstable under 'deformations'). But this is not important for my argument.

Let now $G$ be the Galilei group, an element of which is parameterized by $(R, \boldsymbol{v}, \boldsymbol{a}, b)$, with $R$ a rotation matrix in $S O(3), \boldsymbol{v}$ the boost velocity, $\boldsymbol{a}$ the spatial translation, and $b$ the time translation. Its laws of multiplication and inversion are respectively given by

$$
\begin{aligned}
g_{1} g_{2} & =\left(R_{1}, \boldsymbol{v}_{1}, \boldsymbol{a}_{1}, b_{1}\right)\left(R_{2}, \boldsymbol{v}_{2}, \boldsymbol{a}_{2}, b_{2}\right) \\
& =\left(R_{1} R_{2}, \boldsymbol{v}_{1}+R_{1} \cdot \boldsymbol{v}_{2}, a_{1}+R_{1} \cdot \boldsymbol{a}_{2}+\boldsymbol{v}_{1} b_{2}, b_{1}+b_{2}\right), \\
g^{-1} & =(R, \boldsymbol{v}, \boldsymbol{a}, b)^{-1}=\left(R^{-1},-R^{-1} \cdot \boldsymbol{v},-R^{-1} \cdot(\boldsymbol{a}-\boldsymbol{v} b),-b\right) .
\end{aligned}
$$

We consider the Schrödinger equation for a system of $n$ particles of positions $\boldsymbol{x}_{i}$, masses $m_{i}$, mutual distances $r_{i j}:=\left\|\boldsymbol{x}_{i}-\boldsymbol{x}_{j}\right\|$ which interact via a Galileiinvariant potential $V\left(\left\{r_{i j}\right\}\right)$, so that the Hamilton operator becomes $\mathrm{H}=$ $-\hbar^{2} \sum_{i} \frac{\Delta_{i}}{2 m_{i}}+V$. The Hilbert space is $\mathcal{H}=L^{2}\left(\mathbf{R}^{3 n}, d^{3} \boldsymbol{x}_{1} \cdots d^{3} \boldsymbol{x}_{n}\right)$.

$G$ acts on the space $\{$ configurations $\} \times\{$ times $\} \cong \mathbf{R}^{3 n+1}$ as follows: Let $g=(R, \boldsymbol{v}, \boldsymbol{a}, b)$, then $g\left(\left\{\boldsymbol{x}_{i}\right\}, t\right):=\left(\left\{R \cdot \boldsymbol{x}_{i}+\boldsymbol{v} t+\boldsymbol{a}\right\}, t+b\right)$. Hence $G$ has the obvious left action on complex-valued functions on $\mathbf{R}^{3 n+1}:(g, \psi) \rightarrow \psi \circ g^{-1}$. However, these transformations do not map solutions of the Schrödinger equations into solutions. But as is well known, this can be achieved by introducing an $\mathbf{R}^{3 n+1}$-dependent phase factor (see e.g. [5]). We set $M=\sum_{i} m_{i}$ for the total mass and $\boldsymbol{r}_{c}=\frac{1}{M} \sum_{i} m_{i} \boldsymbol{x}_{i}$ for the center-of-mass. Then the modified transformation, $\mathrm{T}_{g}$, which maps solutions (i.e. curves in $\mathcal{H}$ ) to solutions, is given by

$$
\mathrm{T}_{g} \psi\left(\left\{\boldsymbol{x}_{i}\right\}, t\right):=\exp \left(\frac{i}{\hbar} M\left[\boldsymbol{v} \cdot\left(\boldsymbol{r}_{c}-\boldsymbol{a}\right)-\frac{1}{2} \boldsymbol{v}^{2}(t-b)\right]\right) \psi\left(g^{-1}\left(\left\{\boldsymbol{x}_{i}\right\}, t\right)\right) .
$$

However, due to the modification, these transformations have lost the property to define an action of $G$, that is, we do not have $\mathrm{T}_{g_{1}} \circ \mathrm{T}_{g_{2}}=\mathrm{T}_{g_{1} g_{2}}$. Rather, a straightforward calculation using (7) and (8) leads to

$$
\mathrm{T}_{g_{1}} \circ \mathrm{T}_{g_{2}}=\exp \left(i \xi\left(g_{1}, g_{2}\right)\right) \mathrm{T}_{g_{1} g_{2}},
$$

with non-trivial multiplier exponent

$$
\xi\left(g_{1}, g_{2}\right)=\frac{M}{\hbar}\left(\boldsymbol{v}_{1} \cdot R_{1} \cdot \boldsymbol{a}_{2}+\frac{1}{2} \boldsymbol{v}_{1}^{2} b_{2}\right) .
$$

${ }^{7}$ In General Relativity, where the total mass can be expressed as a surface integral at 'infinity', the issue of mass superselection comes up again; see e.g. [7. 
Although each $\mathrm{T}_{g}$ is a mapping of curves in $\mathcal{H}$, it also defines a unitary transformation on $\mathcal{H}$ itself. This is so because the equations of motion define a bijection between solution curves and initial conditions at, say, $t=0$, which allows to translate the map $\mathrm{T}_{g}$ into a unitary map on $\mathcal{H}$, which we call $\mathrm{U}_{g}$. It is given by

$\mathrm{U}_{g} \psi\left(\left\{\boldsymbol{x}_{i}\right\}\right)=\exp \left(\frac{i}{\hbar} M\left[\boldsymbol{v} \cdot\left(\boldsymbol{r}_{c}-\boldsymbol{a}\right)+\frac{1}{2} \boldsymbol{v}^{2} b\right]\right) \exp \left(\frac{i}{\hbar} \mathrm{H} b\right) \psi\left(\left\{R^{-1}\left(\boldsymbol{x}_{i}-\boldsymbol{a}+\boldsymbol{v} b\right)\right\}\right)$,

and furnishes a ray-representation whose multiplier exponents are given by (11). It is easy to see that the multiplier exponents are non-trivial, i.e., not removable by a redefinition (田). The quickest way to see this is as follows: suppose to the contrary that they were trivial and that hence (4i) holds with $\xi^{\prime} \equiv 0$. Trivially, this equation will continue to hold after restriction to any subgroup $G_{0} \subset G$. We choose for $G_{0}$ the abelian subgroup generated by boosts and space translations, so that the combination $\gamma\left(g_{1}\right)-\gamma\left(g_{1} g_{2}\right)+\gamma\left(g_{2}\right)$ becomes symmetric in $g_{1}, g_{2} \in G_{0}$. But the exponent (11) stays obviously asymmetric after restriction to $G_{0}$. Hence no cancellation can take place, which contradicts our initial assumption.

The same trick immediately shows that the multiplier exponents are inequivalent for different total masses $M$. Hence, by the general argument given in the previous chapter, if $\mathcal{H}^{\prime}$ and $\mathcal{H}^{\prime \prime}$ correspond to Hilbert spaces of states with different overall masses $M^{\prime}$ and $M^{\prime \prime}$, then the requirement that the Galilei group should act on the set of physical states excludes superpositions of states of different overall mass. This is Bargmann's superselection rule.

I criticize these arguments for the following reason: The dynamical framework that we consider here treats 'mass' as parameter(s) which serves to specify the system. States for different overall masses are states of different dynamical systems, to which the superposition principle does not even potentially apply. In order to investigate a possible violation of the superposition principle, we must find a dynamical framework in which states of different overall mass are states of the same system; in other words, where mass is a dynamical variable. But if we enlarge our system to one where mass is dynamical, it is not at all obvious that the Galilei group will survive as symmetry group. We will now see that in fact it does not, at least for the simple dynamical extension which we now discuss.

The most simple extension of the classical model is to maintain the Hamiltonian, but now regarded as function on an extended, $6 n+2 n$ - dimensional phase space with extra 'momenta' $m_{i}$ and conjugate generalized 'positions' $\lambda_{i}$. Since the $\lambda_{i}$ 's do not appear in the Hamiltonian, the $m_{i}$ 's are constants of motion. Hence the equations of motion for the $\boldsymbol{x}_{i}$ 's and their conjugate momenta $\boldsymbol{p}_{i}$ are unchanged (upon inserting the integration constants $m_{i}$ ) and those of the new positions $\lambda_{i}$ are

$$
\dot{\lambda}_{i}(t)=\frac{\partial V}{\partial m_{i}}-\frac{\boldsymbol{p}_{i}^{2}}{2 m_{i}^{2}},
$$


which, upon inserting the solutions $\left\{\boldsymbol{x}_{i}(t), \boldsymbol{p}_{i}(t)\right\}$, are solved by quadrature.

Now, the point is that the new Hamiltonian equations of motion do not allow the Galilei group as symmetries anymore. But they do allow the $\mathbf{R}$ extension $\bar{G}$ as symmetries [5]. Its multiplication law is given by (6), with $\xi$ as in (11). The action of $\bar{G}$ on the extended space of $\{$ configurations $\} \times\{$ times $\}$ is now given by

$$
\begin{aligned}
& \bar{g}\left(\left\{\boldsymbol{x}_{i}\right\},\left\{\lambda_{i}\right\}, t\right)=(\theta, R, \boldsymbol{v}, \boldsymbol{a}, b)\left(\left\{\boldsymbol{x}_{i}\right\},\left\{\lambda_{i}\right\}, t\right) \\
& =\left(\left\{R \boldsymbol{x}_{i}+\boldsymbol{v} t+\boldsymbol{a}\right\},\left\{\lambda_{i}-\left(\frac{\hbar}{M} \theta+\boldsymbol{v} \cdot R \cdot \boldsymbol{x}_{i}+\frac{1}{2} \boldsymbol{v}^{2} t\right)\right\}, t+b\right) .
\end{aligned}
$$

With (6) and (11) it is easy to verify that this defines indeed an action. Hence it also defines an action on curves in the new Hilbert space $\overline{\mathcal{H}}:=$ $L^{2}\left(R^{4 n}, d^{3 n} \boldsymbol{x} d^{n} \lambda\right)$, given by

$$
\overline{\mathrm{T}}_{\bar{g}} \psi:=\psi \circ \bar{g}^{-1},
$$

which already maps solutions of the new Schrödinger equation to solutions, without invoking non-trivial phase factors. This simple transformation law contains the more complicated one (9) upon writing $\overline{\mathcal{H}}$ as a direct integral of vector spaces $\mathcal{H}_{\left\{m_{i}\right\}}$, each isomorphic to our old $\mathcal{H}$. Then, for each $n$-tuple of masses $\left\{m_{i}\right\}$, the new Schrödinger equation reduces to the old one on $\mathcal{H}_{\left\{m_{i}\right\}}$ and (15) restricts to (9) [5].

In the new framework the overall mass, $M$, is a dynamical variable, and it would make sense to state a superselection rule with respect to it. But now $\bar{G}$ rather than $G$ is the dynamical symmetry group, which acts by a proper unitary representation on $\overline{\mathcal{H}}$, so that the requirement that the dynamical symmetry group should act on the space of physical states will now not lead to any superselection rule. Rather, the new and more physical interpretation of a possible superselection rule for $M$ would be that we cannot localize the system in the coordinate conjugate to overall mass, which we call $\Lambda$, i.e., that only the relative new positions $\lambda_{i}-\lambda_{j}$ are observable. 9 (This is so because $M$ generates translations of equal amount in all $\lambda_{i}$.) But this would now be a contingent physical property rather than a mathematical necessity. Note also that in our dynamical setup it is inconsistent to just state that $M$ generates gauge symmetries, i.e. that $\Lambda$ corresponds to a physically non existent degree of freedom. For example, a motion in real time along $\Lambda$ requires a non-vanishing action (for non-vanishing $M$ ), due to the term $\int d t M \dot{\Lambda}$ in the expression for the action.

If decoherence were to explain the (ficticious) mass superselection rule, it would be due to a dynamical instability (as explained in [12]) of those states which are more or less localized in $\Lambda$. Mathematically this effect would be modelled by removing the projectors onto $\Lambda$-subintervalls from the algebra of observables, thereby putting $M$ (i.e. its projectors) into the center of $\mathcal{O}$.

\footnotetext{
${ }^{8}$ A system $\left\{\left(\tilde{\lambda}_{i}, \tilde{m}_{i}\right\}\right)$ of canonical coordinates including $M=\sum_{i} m_{i}$ is e.g. $\tilde{\lambda}_{1}:=$ $\lambda_{1}, \tilde{m}_{1}=M$ and $\tilde{\lambda}_{i}=\lambda_{i}-\lambda_{1}, \tilde{m}_{i}=m_{i}$ for $i=2 \ldots n$. Then $\Lambda=\tilde{\lambda}_{1}$.
} 
Such a non-trivial center should therefore be thought of as resulting from an approximation-dependent idealisation.

\section{Charge Superselection Rule}

In the previous case I said that superselection rules should be stated within a dynamical framework including as dynamical degree of freedom the direction generated by the superselected quantity. What is this degree of freedom in the case of a superselected electric charge and how does it naturally appear within the dynamical setup? What is its relation to the Coulomb field whose rôle in charge-decoherence has been suggested in [7]? In the following discussion I wish to investigate into these questions by looking at the Hamiltonian formulation of Maxwell's equation and the associated canonical quantization.

In Minkowski space, with preferred coordinates $\left\{x^{\mu}=(t, x, y, z)\right\}$ (laboratory rest frame), we consider the spatially finite region $Z=\{(t, x, y, z)$ : $\left.x^{2}+y^{2}+z^{2} \leq R^{2}\right\} . \Sigma$ denotes the intersection of $Z$ with a slice $t=$ const. and $\partial \Sigma=: S_{R}$ its boundary (the laboratory walls). Suppose we wish to solve Maxwell's equations within $Z$, allowing for charged solutions. It is well known that in order for charged configurations to be stationary points of the action, the standard action functional has to be supplemented by certain surface terms (see e.g. 91) which involve new fields on the boundary, which we call $\lambda$ and $f$, and which represent a pair of canonically conjugate variables in the Hamiltonian sense. On the laboratory walls, $\partial \Sigma$, we put the boundary conditions that the normal component of the current and the tangential components of the magnetic field vanish. Then the appropriate boundary term for the action reads

$$
\int_{Z} d t d \omega(\dot{\lambda}+\phi) f
$$

where $\phi$ is the scalar potential and $d \omega$ the measure on the spatial boundary 2sphere rescaled to unit radius. Adding this to the standard action functional and expressing all fields on the spatial boundary by their multipole moments (so that integrals $\int_{\partial \Sigma} d \omega$ become $\sum_{l m}$ ), one arrives at a Hamiltonian function

$$
H=\int_{\Sigma}\left[\frac{1}{2}\left(\boldsymbol{E}^{2}+(\boldsymbol{\nabla} \times \boldsymbol{A})^{2}\right)+\phi(\rho-\nabla \cdot \boldsymbol{E})-\boldsymbol{A} \cdot \boldsymbol{j}\right]+\sum_{l m} \phi_{l m}\left(E_{l m}-f_{l m}\right) .
$$

Here the pairs of canonically conjugate variables are $(\boldsymbol{A}(\boldsymbol{x}),-\boldsymbol{E}(\boldsymbol{x}))$ and $\left(\lambda_{l m}, f_{l m}\right)$, and $E_{l m}$ are the multipole components of $\boldsymbol{n} \cdot \boldsymbol{E}$, where $\boldsymbol{n}$ is the normal to $\partial \Sigma$. The scalar potential $\phi$ has to be considered as Lagrange multiplier. With the given boundary conditions the Hamiltonian is differentiable with respect to all the canonical variables 9 and leads to the following equa-

\footnotetext{
${ }^{9}$ This would not be true without the additional surface term $(16)$. Without it one does not simply obtain the wrong Hamiltonian equations of motions, but
} 
tions of motion

$$
\begin{aligned}
\dot{\boldsymbol{A}} & =\frac{\delta H}{\delta(-\boldsymbol{E})}=-\boldsymbol{E}-\boldsymbol{\nabla} \phi, \\
-\dot{\boldsymbol{E}} & =-\frac{\delta H}{\delta \boldsymbol{A}}=\boldsymbol{j}-\boldsymbol{\nabla} \times(\boldsymbol{\nabla} \times \boldsymbol{A}), \\
\dot{\lambda}_{l m} & =\frac{\partial H}{\partial f_{l m}}=-\phi_{l m}, \\
\dot{f}_{l m} & =-\frac{\partial H}{\partial \lambda_{l m}}=0 .
\end{aligned}
$$

These are supplemented by the equations which one obtains by varying with respect to the scalar potential $\phi$, which, as already said, is considered as Lagrange multiplier. Varying first with respect to $\phi(\boldsymbol{x})$ (i.e. within $\Sigma$ ) and then with respect to $\phi_{l m}$ (i.e. on the boundary $\partial \Sigma$ ), one obtains

$$
\begin{aligned}
G(\boldsymbol{x}) & :=\boldsymbol{\nabla} \cdot \boldsymbol{E}(\boldsymbol{x})-\rho(\boldsymbol{x})=0, \\
G_{l m} & :=E_{l m}-f_{l m}=0 .
\end{aligned}
$$

These equations are constraints (containing no time derivatives) which, once imposed on initial conditions, continue to hold due to the equations of motion. 10

This ends our discussion of the classical dynamical theory. The point was to show that it leaves no ambiguity as to what its dynamical degrees of freedom are, and that we had to include the variables $\lambda_{l m}$ along with their conjugate momenta $f_{l m}$ in order to gain consistency with the existence of charged configurations. The physical interpretation of the $\lambda_{l m}$ 's is not obvious. Equation (20) merely relates their time derivative to the scalar potential's multipole moments on the boundary, which are clearly highly non-local quantities. The interpretation of the $f_{l m}$ 's follow from (23) and the definition of $E_{l m}$, i.e. they are the multipole moments of the local charge-flux $\boldsymbol{n} \cdot \boldsymbol{E}$. In particular, for $l=0=m$ we have

$$
f_{00}=(4 \pi)^{-\frac{1}{2}} Q
$$

none at all! Concerning the Langrangean formalism one should be aware that the Euler-Lagrange equations may formally admit solutions (e.g. with long-ranged (charged) fields) which are outside the class of functions which one used in the variational principle of the action (e.g. rapid fall-off). Such solutions are not stationary points of the action and their admittance is in conflict with the variational principle unless the expression for the action is modified by appropriate boundary terms.

${ }^{10}$ Equation (19) together with charge conservation, $\dot{\rho}+\boldsymbol{\nabla} \cdot \boldsymbol{j}=0$, shows that (22) is preserved in time, and (21) together with the boundary condition that $\boldsymbol{n} \cdot \boldsymbol{j}$ and $\boldsymbol{n} \times(\boldsymbol{\nabla} \times \boldsymbol{A})$ vanish on $\partial \Sigma$ show that (23) is preserved in time. 
where $Q$ is the total charge of the system. Hence we see that the total charge generates motions in $\lambda_{00}$. But this means that the degree of freedom labelled by $\lambda_{00}$ truly exists (in the sense of the theory). For example, a motion along $\lambda_{00}$ will cost a non-vanishing amount of action $\propto Q\left(\lambda_{00}^{\mathrm{final}}-\lambda_{00}^{\text {initial }}\right)$. A declaration that $\lambda_{00}$ really labels only a gauge degree of freedom is incompatible with the inclusion of charges states. Similar considerations apply of course to the other values of $l, m$. But note that this conclusion is independent of the radius $R$ of the spatial boundary 2 -sphere $\partial \Sigma$. In particular, it continues to hold in the limit $R \rightarrow \infty$. We will not consistently get rid of physical degrees of freedom that way, even if we agree that realistic physical measurements will only detect field values in bounded regions of space-time. See [⿶] for more discussion on this point and the distinction between proper symmetries and gauge symmetries.

It should be obvious how these last remarks apply to the statement of a charge superselection rule. Without entering the technical issues (see e.g. [16]), its basic ingredient is Gauss' law (for operator-valued quantities), locality of the electric field and causality. That $Q$ commutes with all (quasi-) local observables then follows simply from writing $Q$ as surface integral of the local flux operator $\boldsymbol{n} \cdot \hat{\boldsymbol{E}}$, and the observation that the surface may be taken to lie in the causal complement of any bounded space-time region. Causality then implies commutativity with any local observable.

In a heuristic Schrödinger picture formulation of QED one represents states $\Psi$ by functions of the configuration variables $\boldsymbol{A}(\boldsymbol{x})$ and $\lambda_{l m}$. The momentum operators are obtained as usual:

$$
\begin{array}{r}
-\boldsymbol{E}(\boldsymbol{x}) \longrightarrow-\mathrm{i} \frac{\delta}{\delta \boldsymbol{A}(\boldsymbol{x})}, \\
f_{l m} \longrightarrow-\mathrm{i} \frac{\partial}{\partial \lambda_{l m}} .
\end{array}
$$

In particular, the constraint (23) implies the statement that on physical states $\Psi$ we have

$$
\hat{Q} \Psi=-\mathrm{i} \sqrt{4 \pi} \frac{\partial}{\partial \lambda_{00}} \Psi .
$$

This shows that a charge superselection rule is equivalent to the statement that we cannot localize the system in its $\lambda_{00}$ degree of freedom. Removing by hand the multiplication operator $\lambda_{00}$ (i.e. the projectors onto $\lambda_{00}$-intervals) from our observables clearly makes $Q$ a central element in the remaining algebra of observables. But what is the physical justification for this removal? Certainly, it is valid FAPP if one restricts to local observations in space-time. To state that this is a fundamental restriction, and not only an approximate

${ }^{11}$ Clearly all sorts of points are simply sketched over here. For example, charge quantization presumably means that $\lambda_{00}$ should be taken with a compact range, which in turn will modify $(26)$ and $(27)$. But this is irrelevant to the point stressed here. 
one, is equivalent to saying that for some fundamental reason we cannot have access to some of the existing degrees of freedom, which seems at odds with the dynamical setup. Rather, there should be a dynamical reason for why localizations in $\lambda_{00}$ seem FAPP out of reach. Again, if decoherence is to explain the phenomena of an apparent charge superselection rule, localization in $\lambda_{00}$ must be highly unstable against dynamical decoherence.

Regarding the charge superselection rule, Erich Joos asked the following question in section 3.2 of his contribution to this volume: "What is the quantum physical rôle of the Coulomb field". In some sense the analysis given here is also meant to shed some light on this question. We have seen the necessity to include the non-local canonical variables $\lambda_{l m}, f_{l m}$ and discussed their relation with the $\frac{1}{r^{2}}$ - part of the electric field. Clearly, the arguments given here are neither complete nor rigorous in any sense. What they suggest is to relate the $\lambda_{l m}$ degrees of freedom to the precise infrared structure of QED, for example along the lines of [20] 21] [9. Eventually this raises the question of how to fully describe the state space of QED, which is known to be a notoriously difficult problem [2].

Also, from my presentation it did not become apparent why charge plays such a particular rôle among the other superselection operators $\hat{f}_{l m}$ associated with the higher multipole moments of the asymptotic flux distribution. This question is further analyzed in [2] and from a more concrete latticecalculational point of view in [13]. 


\section{References}

1. Bargmann, V. (1964): Note on Wigner's Theorem on Symmetry Operations. Jour. Math. Phys. 5, 862-868

2. Buchholz, D. (1982): The Physical State Space of Quantum Electrodynamics. Commun. Math. Phys. 85, 49-71

3. Galindo, A., Pascual, P. (1990): Quantum Mechanics I (Springer, Berlin)

4. Giulini, D. (1995): Asymptotic Symmetry Groups of Long-Ranged Gauge Configurations. Mod. Phys. Lett A 10, 2059-2070

5. Giulini, D. (1996): On Galilei Invariance and the Bargmann Superselection Rule. Ann. Phys. (NY) 249, 222-235

6. Giulini, D. (1995): Quantum Mechanics on Spaces with Finite Fundamental Group. Helv. Phys. Acta 68, 438-469

7. Giulini, D., Kiefer, C. Zeh, H.D. (1995): Symmetries, Superselection Rules, and Decoherence. Phys. Lett. A 199, 291-298

8. Giulini, D, Joos, E., Kiefer, C., Kupsch, J., Stamatescu, I.-O., Zeh, H.-D. (1996): Decoherence and the Appearance of a Classical World in Quantum Theory, (Springer, Berlin)

9. Gervais, J.L., Zwanziger, D. (1980): Derivation From First Principles of the Infrared Structure of Quantum Electrodynamics. Phys. Lett. B 94, 389-393

10. Jauch, J.M. (1960): Systems of Observables in Quantum Mechanics. Helv. Phys. Acta 33, 711-726

11. Jauch, J.M., Misra,B. (1961): Supersymmetries and Essential Observables. Helv. Phys. Acta 34, 699-709

12. Joos, E. (1999) Elements of Environmental Decoherence, contribution to this volume.

13. Kijowski, J., Rudolph, G., Thielmann, A. (1997): Algebra of Observables and Charge Superselection Sectors for QED on the Lattice. Commun. Math. Phys. 188, 535-564

14. von Neumann, J. (1932): Mathematische Grundlagen der Quantenmechanik, (Springer, Berlin)

15. Raghunathan, M.S. (1994): Universal Central Extensions. Rev. Math. Phys. 6, 207-225

16. Strocchi, F, Wightman, A.S. (1974): Proof of the Charge Superselection Rule in Local Relativistic Quantum Field Theory. Jour. Math. Phys. 15, 2198-2224; Erratum Ibid 17 (1976), 1930-1931

17. Wick, G.C., Wightman, A.S., Wigner, E.P. (1952): The Intrinsic Parity of Elementary Particles. Phys. Rev. 88, 101-105

18. Wightman, A.S., Glance N. (1989): Superselection Rules in Molecules. Nucl. Phys. B (Proc. Suppl.) 6, 202-206

19. Wightman, A.S. (1995): Superselection Rules; Old and New. Il Nuovo Cimento $110 \mathrm{~B}, 751-769$

20. Zwanziger, D. (1976): Physical States in Quantum Electrodynamics. Phys. Rev. D 14, 2570-2589

21. Zwanziger, D. (1978): Gupta-Bleuler and Infrared-Coherence Subsidiary Conditions. Phys. Rev. D 18, 3051-3057 